\title{
Caractérisation de neuf échantillons de farine de maïs Zea mays (L.) vendus sur les marchés d'Adjamé, Yopougon et Abobo en Côte d'Ivoire
}

\author{
* 1 Mme DEDI née KY Juliette, ${ }^{2}$ GBEHE Stéphane, ${ }^{3}$ YOUO Dékpassi Clément \\ 1 Laboratoire de Biologie et Amélioration des Productions Végétales, Université Nangui ex. université d'Abobo-Adjamé, \\ UFR-SN, 074306 33, 01 BP 8133 Abidjan 01, Côte d'Ivoire mmededijuliette@yahoo.fr \\ 2 Laboratoire de Biologie et Amélioration des Productions Végétales, Université Nangui ex. université d'Abobo-Adjamé), \\ UFR-SN, 575403 26, 02 BP 801 Abidjan 02, Côte d'Ivoire stephanegbehe@gmail.com \\ 3 Laboratoire de Biologie et Amélioration des Productions Végétales, Université Nangui Abrogoua, UFR-SN, 485966 76, \\ 02 BP 801 Abidjan 02, Côte d'Ivoire \\ Correspondance email : ydclement@gmail.com
}

Original submitted in on 9th June 2017. Published online at www.m.elewa.org on $31^{\text {st }}$ July 2017

https://dx.doi.org/10.4314/jab/v115i1.3

\section{RÉSUMÉ}

Objectif : Le présent travail a pour objectif de caractériser la flore de neuf échantillons de farine de maïs Zea mays (L.)] achetés sur trois marchés d'Abidjan (Adjamé, Yopougon, Abobo).

Méthodologie et résultats : A l'issue de l'inoculation du milieu de culture PDA avec les différentes suspensionsdilutions de farine, cinq genres de champignons ont été isolés et identifiés à des pourcentages variés. $\mathrm{A}$ Adjamé, Aspergillus niger est respectivement présent à 41,45 et $33,33 \%$; Fusarium sp. à $33,35,66,66 \%$; $36,84 \%$ à Abobo chez la 1 ère vendeuse et 33,33\%, $25 \%$ respectivement chez la 1 ère et la 3ème vendeuse à Yopougon. A yopougon Penicillium sp. est présent sur la 1ère (16,66 \%) et la 2ème $(40 \%)$ farine et sur la 1ère $(21,05 \%)$ farine à Abobo. Aspergillus flavus a été isolé sur les farines $2(10 \%)$ et $1(31,57 \%)$ respectivement à Adjamé et à Abobo. Botrytis sp. était présent à Adjamé, Yopougon et Abobo respectivement sur les farines 1 et $2(25 \%, 10 \%) ; 3(25 \%)$ et sur $1(10,52 \%)$. La présence de ces champignons a entrainé la détérioration des farines en provenance d'Adjamé et de la farine 1 achetée à Abobo. En fonction des marchés, le pourcentage moyen d'apparition le plus élevé est attribué à Fusarium sp. (44,88 \%) à Adjamé, ensuite à Mucor sp. $(28,33$ $\%)$ à Yopougon puis à Aspergillus flavus (10,52 \%) à Abobo.

Conclusion et application des résultats : Les farines achetées sur les trois marchés renferment toutes des champignons parmi lesquels figurent les genres Aspergillus, Penicillium et Fusarium reconnus comme producteurs de mycotoxines. Ces derniers sont peu volatiles, très stables à l'acidité et à la chaleur. II est donc important que des mesures d'hygiène rigoureuse soient observées au moulin, au cours du séchage et de la conservation de la farine afin que le consommateur soit à l'abri de toute intoxication.

Mots clés : Farine, état sanitaire, champignons, suspension dilution, marchés 


\section{ABSTRACT}

Objective : The aim of this work is to characterize the fungal flora of nine samples of maize flour [Zea mays (L.)] purchased from three markets in Abidjan (Adjamé, Yopougon, Abobo).

Methodology and results: At the end of the inoculation of the PDA culture medium with the different flour dilution suspensions, five kinds of fungi were isolated and identified at various percentages. At Adjamé, Aspergillus niger is respectively present at 41,45 and $33.33 \%$; Fusarium sp. To $33,35,66.66 \% ; 36.84 \%$ in Abobo in the 1st seller and 33.33\%, 25\% respectively in the 1st and 3rd in Yopougon. A yopougon Penicillium sp. Is present on the $1 \mathrm{st}(16.66 \%)$ and the $2 \mathrm{nd}(40 \%)$ flour and on the $1 \mathrm{st}(21.05 \%)$ flour in Abobo. Aspergillus flavus was isolated on flour $2(10 \%)$ and $1(31.57 \%)$ respectively at Adjame and Abobo. Botrytis sp. Was present in Adjamé, Yopougon and Abobo respectively on flour 1 and 2 (25\%, 10\%); $3(25 \%)$ and $1(10.52 \%)$. The presence of these fungi leads to the deterioration of the flours from Adjamé and the flour 1 purchased from Abobo. Depending on the markets, Fusarium sp. (44.88\%) to Adjamé, then to Mucor sp. (28.33\%) in Yopougon and then in Aspergillus flavus (10.52\%) in Abobo.

Conclusion and application of results: The flours purchased in the three markets all contain mushrooms, including the genera Aspergillus, Penicillium and Fusarium, which are recognized as producers of mycotoxins. The latter are not very volatile, very stable to acidity and heat. It is therefore important that rigorous hygiene measures be observed at the mill, during the drying and storage of the flour, so that the consumer is protected from any poisoning.

Keywords: Flour, status, mushrooms, suspension dilution, markets

\section{INTRODUCTION}

Le maïs [Zea mays (L.)] est une céréale cultivée dans des conditions très variées allant du climat tropical au climat tempéré, (Hoopen et Maïga, 2012). Selon Akanvou et al., (2006a) c'est la première céréale ( $41 \%$ ) cultivée dans le monde en termes de quantité de production et de surface devant le blé $(40 \%)$ et le riz (9\%). La production de maïs en Afrique est estimée à plus de 70 millions de tonnes pour une superficie de 34 millions d'hectares (Harold et Tabo, 2015). Selon ces derniers, il compose presque la moitié des apports en calories, en protéines en Afrique Orientale et Australe et un cinquième en Afrique occidentale. Ils estiment également que plus de 300 millions de personnes en Afrique sub-saharienne dépendent du maïs comme source de subsistance. Les zones consacrées à la production de maïs ont augmenté de manière considérable dans les régions d'Afrique subsaharienne depuis 1961 et la Côte d'Ivoire fait partie des 20 premiers pays qui produisent $96 \%$ du maïs (FAOSTAT, 2015). En Côte-d'Ivoire, le maïs occupe une place de choix aussi bien dans les activités agricoles, dans l'alimentation des populations ivoiriennes que dans l'alimentation animale. Le maïs constitue l'aliment de base de nombreuses populations ivoiriennes et représente $68 \%$ de la production totale de céréales nationale (MINAGRI, 2010). Il est cultivé dans différentes régions du pays à savoir le Poro, le Hambol, la Bagoué, le Haut Sassandra, le Folon, le Kabadougou, le Worodougou et le Beré. En provenderie, il intervient dans l'alimentation animale (volailles, porcs, bovins) (Hoopen et Maïga, 2012). Cependant, il existe un certain nombre de contraintes liées à la production du maïs. Elles peuvent se résumer à l'insuffisance et à l'irrégularité des pluies, à la dégradation et à l'appauvrissement des sols, à l'abondance des adventices (mauvaises herbes) aux maladies d'origine fongique (Heiminthosporium maydis et Curvilariose sp.) et au coût élevé des intrants. Le maïs est consommé à $92 \%$ sous forme de farine et frais à raison de $28,5 \mathrm{~kg} /$ habitant/an (Countrystat, 2013) et les produits alimentaires de seconde transformation à base de farine de maïs sont entre autres le «toh», les beignets, les galettes et la bouillie de maïs. La récolte et le stockage sont des étapes généralement identifiées de la contamination des grains qui peut se faire par: la terre, les impuretés, la poussière générée à la récolte, la présence éventuelle de foyers d'infection dans les moyens de transport et les silos (notamment dus à la présence d'humidité, d'insectes, de rongeurs ou 
d'oiseaux). Les champignons principalement représentés sont les genres Fusarium, Alternaria, Cladosporium, Aspergillus et Penicillium (Mehrez, 2008). Cependant, au cours du processus de mouture, des sources de contamination existent. On peut toutefois citer : les foyers d'infection localisés dans les circuits (pieds d'élévateurs, tuyauteries, zones de stagnation de produit humide) notamment dus au développement de moisissures, les contaminations dues à la présence d'insectes, de rongeurs ou d'oiseaux dans les locaux et les contaminations d'origine humaine dues à l'hygiène des opérateurs et à la gestion des gâchis (Souply, 2008). Makambila et Loubana, (1990) ont réalisé au Congo des isolements à partir du maïs prélevés

\section{MÉTHODES}

Matériel végétal : Le matériel végétal, objet de cette étude, est la farine de maïs (ZEA Mays) achetée dans les marchés d'Adjamé, de Yopougon et d'Abobo au mois de janvier 2016. Elle a été obtenue après la mouture des graines de couleur jaune dont un échantillon nous a été fourni par chacune des vendeuses. La variété EV8728 (corné et semi denté) pour Adjamé, F8128 Long (100120, corné/denté) pour Yopougon et EV8766-SRMRP (semi denté, riche en protéines) à Abobo. L'identification des graines a été faite par le Centre National de Recherche Agronomique (CNRA) de Korhogo.

Préparation des échantillons de farine : Elle a été faite au laboratoire dans la zone stérile du bec Bunsen. Les échantillons ont été homogénéisés puis passés au travers d'un tamis métallique préalablement aseptisé par dans les silos et de petites quantités de farine à différents stades de la transformation, jusqu'au produit fini. Toutes les cultures obtenues à partir de des ensemencements ont mis en évidence la présence de microorganismes. Des investigations mycologiques effectuées au Nigeria par Adebajo et al., (1994), ont démontré des niveaux de contaminations élevés dans des gâteaux de maïs. Selon Lugauskas et al., (2006), la fréquence de contamination de la farine de maïs par les genres Aspergillus et Penicillium est supérieure à $50 \%$. L'objectif de ce travail est d'évaluer la qualité sanitaire des farines de maïs vendues sur les marchés de trois communes d'Abidjan.

passage rapide dans la flamme du bec Bunsen. Débarrassés des impuretés, les produits du tamisage ont été conservés dans des pots en plastiques propres et placés dans un lieu frais et sec.

Détermination de la teneur en eau de la farine : La méthode d'analyse est la dessiccation à l'étuve. Cinq grammes $(P)$ de farine sont introduits dans un vase métallique taré après dessiccation à l'étuve et refroidissement dans le dessiccateur. Chaque vase couvert est ensuite mis à l'étuve pendant $1 \mathrm{H} 30$ minutes à $130^{\circ} \mathrm{C}$. L'échantillon (reste couvert) a été refroidi dans le dessiccateur à l'abri de l'humidité ambiante puis pesé $\left(P_{1}\right)$.Trois répétitions ont été faites pour chaque échantillon de farine. La teneur en eau ou le pourcentage d'humidité est déterminé par la formule qui suit :

$$
T E=\frac{P-P 1}{P} \times 100
$$

\section{Isolement et identification des champignons}

Préparation du milieu de culture Potato Dextrose Agar (PDA) : Vingt grammes d'agar-agar ont été dissous dans $300 \mathrm{ml}$ d'eau distillée puis homogénéisée. Deux cent grammes de pomme de terre épluchée ont été pesés, lavés, découpés en morceaux et mis à bouillir dans $300 \mathrm{ml}$ d'eau distillée à $100^{\circ} \mathrm{C}$ pendant 20 à $25 \mathrm{mn}$. L'eau de cuisson a été filtrée, recueillie et incorporée au $300 \mathrm{ml}$ de la solution d'agar-agar. Le volume final du mélange a été ajusté à $1 \mathrm{~L}$ avec de l'eau distillée et

\section{TE : Teneur en Eau}

P : Prise d'essai

P1 : Poids du résidu sec

autoclavé à $121^{\circ} \mathrm{C}$ à la pression de 1,4 bar pendant 15 $\mathrm{mn}$.

Préparation des suspensions dilution et ensemencement: La préparation des dilutions a consisté à ajouter $10 \mathrm{~g}$ de farine tamisée, broyée finement à sec dans un mortier muni d'un pilon en porcelaine à $100 \mathrm{ml}$ d'eau stérile. Cette suspension est homogénéisée sur un agitateur magnétique Riihromag $\mathrm{M}$ 13 à la vitesse maximale pendant 10 minutes et a constitué la dilution 10-1. Des prélèvements successifs de 
$1 \mathrm{ml}$ dans cette suspension, puis dans les suivantes ajoutés chaque fois à $9 \mathrm{ml}$ d'eau stérile ont constitué les dilutions $10^{-2}$ jusqu'à $10^{-6}$. Ces différents prélèvements ont été effectués avec des pipettes stériles qui sont changées à chaque dilution. Un millilitre de chaque dilution a été étalé par boîte de Pétri sur le milieu gélosé, refroidi et durci. Trois répétitions par dilution ont été effectuées.

Isolats fongiques: Après incubation, une mycoflore variée s'est développée. Afin de purifier les souches et les maintenir en culture pure, deux à trois repiquages successifs sur milieu PDA ont été effectués. Une fois purifiées, les souches sont identifiées sur la base de leurs caractères culturaux et morphologiques (Barnett et Hunter, 1972 ; Champion, 1997). Ces données ont permis la détermination du genre de chaque souche.

Pourcentage d'apparition de chacun des champignons (Mouria et al., 2013): Le calcul du pourcentage s'est fait selon la formule suivante :

$$
\% \text { apparition }=\frac{\text { NAC }}{\text { NTATC }}
$$

$\%=$ pourcentage $;$ NAC = Nombre d'Apparition du Champignon ;

NTATC $=$ Nombre Total d'Apparition de Tous les Champignons.

Le dénombrement des champignons : L'analyse de la mycoflore a été conduite selon la technique des suspensions - dilutions.

Analyse statistique : Une analyse de la variance à deux critères de classification (ANOVA2) est faite pour comparer la variance des moyennes de la teneur en eau

\section{RÉSULTATS}

Teneur en eau de la farine de maïs de différentes localités: Quantitativement, l'eau est le deuxième constituant de la farine. On peut s'en étonner car sa présence n'est détectable ni à l'œil, ni au toucher. La teneur en eau des farines est comprise entre 12,12 et $18,02 \%$. La teneur la plus élevée est obtenue à Adjamé chez la $2^{\mathrm{e}}$ vendeuse et la plus faible à Yopougon des échantillons de farine et leur localité. Lorsqu'une différence significative a été observée entre les moyennes de ces paramètres, le test de Newman-Keuls a été appliqué au seuil de $5 \%$, pour la séparation des moyennes grâce au logiciel $R$ version 3.3.1.

également chez la $2^{\mathrm{e}}$ vendeuse. Chez les 1 ère vendeuses par commune, Abobo a la plus forte teneur en eau. Chez

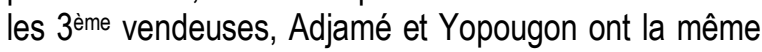
teneur en eau. Les teneurs en eau pour Adjamé, Yopougon et Abobo sont comprises respectivement entre 15,08 et $18,02 \%, 12,12$ et $15 \%$ et 13,08 et $17 \%$ (Tableau 1).

Tableau 1: Teneur en eau des échantillons par commune

\begin{tabular}{|l|c|c|c|}
\hline \multicolumn{4}{|c|}{ Teneur en eau (\%) } \\
\hline Marchés Vendeuses & Adjamé & Yopougon & Abobo \\
\hline 1 & $16 \pm 2,01^{\mathrm{a}}$ & $15 \pm 1,2^{\mathrm{a}}$ & $17 \pm 1,66^{\mathrm{a}}$ \\
\hline 2 & $18,02 \pm 1,05^{\mathrm{b}}$ & $12,12 \pm 2,01^{\mathrm{b}}$ & $13,08 \pm 1,16^{\mathrm{b}}$ \\
\hline 3 & $15,05 \pm 1,52^{\mathrm{a}}$ & $15 \pm 1,4^{\mathrm{a}}$ & $14,06 \pm 2,05^{\mathrm{b}}$ \\
\hline 4 Moyenne/marché & 16,35 & 14,04 & 14,71 \\
\hline
\end{tabular}

Dans la même colonne, les chiffres suivis de la même lettre sont statistiquement identiques au seuil de 5 \% (Newman-Keul).

Champignons isolés et identifiés: L'analyse de la mycoflore naturelle des différents échantillons de farine de maïs a pu mettre en évidence Aspergillus flavus, $A$. niger, Botrytis sp., Fusarium sp., Mucor sp. et Penicillium sp.. Cette identification est basée sur une analyse macro et microscopique des isolats fongiques sur milieu Potato Dextrose Agar (PDA). L'observation microscopique s'est faite entre lame et lamelle. Les caractères microscopiques (morphologie du mycélium et sa coloration, forme et coloration des spores ou conidies, présence ou non de structures de conservation) basés sur les clés d'identification sont décrits. Cette identification a été faite à l'aide de clés d'identification de Barnet et Hunter (1972), et Champion (1990). Le tableau 2 donne le pourcentage d'apparition des différents champignons. Aspergillus niger, Botrytis sp. et Fusarium 

sur les marchés d'Adjamé, Yopougon et Abobo en Côte d'Ivoire

sp. sont présents sur tous les marchés mais pas sur toutes les farines. Aspergillus niger est plus présent sur les farines $1(41 \%)$ et $2(45 \%)$ à Adjamé et très faible dans la farine $1(3,75 \%)$ à Abobo. Fusarium sp. et Mucor $\mathrm{sp}$. sont respectivement très présents sur la farine 3 $(66,66 \%)$ à Adjamé et la farine $2(60 \%)$ à Yopougon. Quant à Aspergillus flavus il est uniquement présent à Adjamé sur la farine 2 (10\%) (Tableau 2).

Tableau 2 : Pourcentage d'apparition des champignons

\begin{tabular}{|c|c|c|c|c|}
\hline \multicolumn{5}{|c|}{ \% d'apparition des champignons } \\
\hline & Adjamé & Yopougon & \multicolumn{2}{|c|}{ Abobo } \\
\hline Champignons & 123 & 123 & \multicolumn{2}{|c|}{123} \\
\hline Aspergillus flavus & $-10-$ & $-\quad-\quad-$ & 31,57 & - \\
\hline Aspergillus niger & $41 \quad 45 \quad 33,33$ & $50-25$ & $-\quad-$ & - \\
\hline Botrytis sp & $25 \quad 10 \quad-$ & $-\quad-25$ & 10,52 & - \\
\hline Fusarium $\mathrm{sp}$ & $\begin{array}{lll}33 & 35 & 66,66\end{array}$ & $33,33-25$ & 36,84 & . \\
\hline Mucor sp & $-\quad-$ & -6025 & - & - \\
\hline Penicillium sp & $-\quad-$ & $16.6640-$ & 21.05 & \\
\hline
\end{tabular}

\section{DISCUSSION}

Les teneurs en eau des farines des vendeuses 1 et 2 d'Adjamé et celle de la farine de la vendeuse 1 d'Abobo sont supérieures à $15 \%$. Selon Chene, (2001), la teneur en eau qui est un paramètre important se situe généralement entre 13 et $15 \%$ pour une bonne conservation de la farine. Trop d'humidité est cause d'altération de la farine. II a été montré qu'il y a une relation entre l'humidité dans l'aliment et la capacité de croissance microbienne (Nguyen, 2007). Ces trois farines ne sont pas conformes à la réglementation. En dehors de ces trois farines, toutes les autres, tous marchés confondus ont une teneur allant de 12,12 à 15,02 \%. Probablement, elles ont été séchées plus longtemps ce qui a entrainé une importante évaporation de l'eau pour une bonne conservation. Bien séchée, la farine de maïs donne des mets très agréables à déguster lorsque celle-ci est cuite. De façon générale, l'ensemble des farines en notre possession, qui étaient destinées à la consommation, renferment des champignons que sont Aspergillus niger, Aspergillus flavus, Fusarium sp. Botrytis sp., Penicillium sp. et Mucor sp. à l'exception de Botrytis sp. et Fusarium sp., Aspergillus niger, Aspergillus flavus, Penicillium sp., Mucor sp. et Rhizopus sp. qui ont été identifiés à partir des isolats fongiques associés à la farine de mil ((Mehrez, 2008). A Adjamé, Aspergillus niger $(39,77 \%)$, Botrytis sp. (11,66 \%) et Fusarium sp. $(44,88$ $\%$ ) ont un pourcentage moyen d'apparition des champignons sur les trois farines supérieur à ceux de Yopougon et d'Abobo. II se trouve qu'à Yopougon, ce sont les pourcentages d'apparition de Mucor sp. (28,33 $\%$ ) et Penicillium sp. (18,88\%) qui sont supérieurs à ceux d'Adjamé et d'Abobo. Cependant, à Abobo, Aspergillus flavus a son pourcentage d'apparition moyen supérieur à ceux d'Adjamé et de Yopougon. La présence d'une telle mycoflore typique de stockage (Czerwieci et al., 2002 ; Cast, 2003), nous laisse supposer que la contamination a probablement eu lieu au cours du stockage des grains de maïs. Selon Tabuc, (2007), la contamination a probablement eu lieu avant la récolte, au champ, au cours du séchage, du stockage et après transformation des graines. Les céréales représentent une matière première importante pour l'alimentation. Elles sont transformées en farine à partir desquelles sont élaborées de nombreux aliments finis (Tabuc, 2007). Ces produits comme les aliments composés pour les animaux, peuvent être contaminés soit par les spores qui se trouvent initialement dans les céréales soit plus tard pendant le stockage ou lors des manipulations de la farine pendant la vente sur les marchés. Des investigations mycologiques effectuées au Nigeria par Adebajo et al., (1994), ont démontré des niveaux de contaminations élevés dans des gâteaux de maïs. Selon Lugauskas et al., (2006), la fréquence de contamination de la farine de maïs par les genres Aspergillus et Penicillium est supérieure à $50 \%$. Au cours de notre travail, les pourcentages moyens de contamination par ces deux champignons n'atteignent pas les $50 \%$. Les champignons qui ont contaminé les farines appartiennent aux genres Aspergillus, Fusarium, Botrytis, Penicillium et Mucor. Parmi ces derniers, Aspergillus, Fusarium et Penicillium sont producteurs de mycotoxines qui sont des métabolites secondaires peu labiles et souvent actifs à très faibles doses. Elles diffusent dans le substrat qu'elles contaminent même après la destruction du champignon responsable de leur production (Siou, 2013). Les métabolites secondaires sont des inhibiteurs de synthèse 
protéique des cellules eucarytes (Cumagun et al., 2004) et de l'activation des gènes de défense de la plante (Wagacha et Muthomi, 2007). L'Organisation des Nations Unies pour I'Alimentation et l'Agriculture estime que 25\% des produits agricoles dans le monde sont contaminés par des mycotoxines, entraînant des pertes économiques importantes (Measuring, 2007). Les différentes farines en notre possession renferment des champignons (Aspergillus niger, Aspergillus flavus, Fusarium sp., Mucor sp. et Penicillium sp.) susceptibles de produire des mycotoxines dont les plus connues sont les aflatoxines, les fumonisines, l'ochratoxine, la zéaralénone, les trichothécènes et les alcaloïdes de l'ergot (Miller, 1998) si les conditions sont réunies [Castegnaro et PfohlLeszkowicz , (2002) ; Pfohl-Leszkowicz, (2001)]. La présence de champignons ochratoxinogènes tels que Aspergillus niger (Abarca et al., 1994) et Penicillium (Meri

\section{CONCLUSION}

La Côte d'Ivoire, en raison de son climat, des habitudes alimentaires particulières de sa population, des conditions de stockage et de transformation des denrées alimentaires, est un pays favorable au développement des champignons toxinogènes contaminants fréquents de nombreux substrats végétaux telle que la farine de maïs et de certains produits d'origine animale. Leur présence peut améliorer les qualités organoleptiques du produit ou, au contraire, l'altérer et conduire à l'accumulation de métabolites secondaires toxiques : les mycotoxines. La teneur en eau élevée a probablement favorisé le

\section{RÉFÉRENCES BIBLIOGRAPHIQUES}

Abarca ML, Bragulat MR, Castella G, Cabanes FJ, 1994. Ochratoxin A production by strains of Aspergillus niger var niger. Appl Environ Microbiol. 60: 2650- 2652.

Adebajo LO, Idowu AA, Adesanya OO, 1994. Mycoflora, and mycotoxins production in Nigerian corn and corn-based snacks, Mycopathologia, 126 (3); 183-192.

Akanvou L, Akanvou R, Toto K, 2006a. Effets des variétés de maïs et de légumineuses dans la lutte contre Striga hermonticaen zone de savane en Côte d'Ilvoire, agronomie africaine18(1) : 1321.

Barnett HL, Hunter Barry B, 1972. Illustrated genera of imperfecti fungi. Third edition. Burgess Publishing Company. Minneapolis, USA) 241.

CAST (Council for Agricultural Science and Technology), 2003. Ames, lowa, USA Mycotoxins : Risks in et al., 2005), plaide en faveur d'une contamination en OTA. Ce qui a été démontré avec des échantillons stockés de farine de mil (Mehrez, 2008). La stabilité des mycotoxines leur permet d'entrer dans la chaîne alimentaire tout en conservant leurs propriétés toxiques (Saint-Cyr, 2013). Certaines d'entre elles seraient également impliquées dans l'apparition de maladies chez l'Homme (CAST 2003). Chez les animaux de rente, tels que les bovins laitiers, les porcs et les volailles, la contamination des aliments composés par les mycotoxines entraine la réduction de la consommation et le taux de reproduction altère la résistance aux maladies infectieuses, réduit l'efficacité de la vaccination et induit des dommages pathologiques dans le principal organe cible le foie mais aussi dans d'autres organes comme l'intestin et la rate (Ramakrishna et al., 1989).

développement de champignons qui sont de surcroit de potentiels (Aspergillus niger, Aspergillus flavus, Fusarium $\mathrm{sp}$. et Penicillium sp.) producteurs de mycotoxines dans quatre des échantillons de farine en notre possession qui ont été probablement à la base de la détérioration de ces farines. Nous courons des risques en consommant la farine moulue, séchée et exposée à l'air ambiant de nos marchés ou elle est vendue tassée dans des bassines métalliques quelques fois rouillées et servie avec la main non protégée.

plant, animal and human systems, Task force report No. $139199 \mathrm{p}$.

Castegnaro M, Pfohl-Leszkowicz A,(2002). Les mycotoxines : contaminants omniprésents dans l'alimentation animale et humaine dans la sécurité alimentaire. Moll \& Moll (eds) Lavoisier. Tec \& Doc, $5 p$.

Champion $R$, 1997. Identifier les champignons transmis par les semences. INRA (Institut National de la Recherche Agronomique) 147, rue de I'Université, 75338 Cedex 07, 398p.

Chene A, 2001. La farine. Journal de l'ADRIANOR, 26, 38.

Countrystat 2013. http/l : www.countrystat. org/civ/cont/pxwebquery/ma/107spd080/fr. 17 septembre 2016.

Cumagun CJR, Bowden RL, Jurgenson JE, Leslie JF, Miedaner T, 2004. Genetic mapping of pathogenicity and aggressiveness of Gibberella 
zeae (Fusarium graminearum) toward wheat. Phytopathology 94,520-526.

Czerwiecki L, Czajkowska D, Witkowska-Gwiazdowska $A$, 2002. Ochratoxin A and fungal flora in Polish cereals from conventional and ecological farms. Food Addit. Contam. Vol. 19(5):470-477.

FAOSTAT 2015. http://Faostat.fao.org. 18 mai 2016.

Harold M, Tabo R, 2015. Les cultures céréalières : riz, maïs, millet, sorgho et blé document (37) : 1-6.

Lugauskas A, Raila A, Railiene M, Raudoniene V, 2006. Toxic micromycetes in grain raw material during its processing, Ann. Agric. Environ. Med., 13, 147-161.

Measuring 2007 the economic impacts of Fusarium toxins in animal feeds. Animal Feed Science and Technology, 137(3): p. 363-374.

Makambila C, Loubana PM, 1990. La post -récolte en Afrique. Actes du Séminaire International tenu à Abidjan Côte d'Ivoire. FOUA-BI M, Bernard JR. Philogène. 276p.

Mehrez A, 2008. Effets du radiotraitement par les rayonnements gamma sur la décontamination et la cytotoxicité d'une mycotoxine : l'Ochratoxine A. Université 7 novembre à Carthage Tunisie. Faculté des Sciences de Bizerte Département des Sciences de la vie. Master Sciences de L'Environnement 145p.

Meri K, Marika J, Aldo R, 2005. The effect of substrate on mycotoxin production of selected Penicillium strains. International Journal of Food Microbiology. 99 :207-214.

Miller J, 1998. Global significance of mycotoxins. Mycotoxins and Phycotoxins- Developments in Chemistry, Toxicology and Food Safety Alaken Inc., Ford Collins, Colorado, p. 3-15.

MINAGRI (Ministère de l'agriculture), 2010. Production céréalière en Côte-d'Ivoire. Document $1 \mathrm{du}$ séminaire national sur les potentialités et les contraintes de la production céréalière en Côted'Ivoire, 15-20.

Mouria B, Ouazzani-Touhami A, Douira A, 2013.Isolement et identification de la mycoflore du compost des déchets urbains solides, Nature \& Technologie, C- Sciences de l'Environnement, $n^{\circ}$ 09. P. 13- 28.

Nguyen MT, 2007. Identification des espèces de moisissures, potentiellement productrices de mycotoxines dans le riz commercialisé dans cinq provinces de la région centrale du Viêtnam. Des conditions pouvant réduire la production des mycotoxines. Thèse / Institut National
Polytechnique de Toulouse pour le titre de Docteur. 147p.

Pfohl-Leszkowicz A, 2001. Définition et origins des mycotoxines dans l'alimentation : évaluation et gestion du risque. Tec \& Doc, PP. 3-14.

Ramakrishna Y, Bhat RV, Ravindranath V, 1989. Production of deoxynivalenol by Fusarium isolates from samples of wheat associated with a human mycotoxicosis outbreak and from sorghum cultivars. Applied and Environmental Microbiology 55 : 2619-2620.

Saint-Cyr M, 2013. Impact des Mycotoxines sur le Microbiote Intestinal Humain, cas particulier du Déoxynivalénol Thèse / Université de Rennes 1 sous le sceau de l'Université Européenne de Bretagne pour le grade de Docteur de L'Université de Rennes 1 Mention: Biologie et Sciences de la Santé: Vie, Agro, Santé présentée par 196p.

Siou D, 2013. Développement épidémique de la fusariose des épis de blé et conséquences des interactions entre espèces du complexe fusarien. Sciences agricoles. Université Paris Sud - Paris XI. 196p.

Souply F, 2008. Analyses microbiologiques des farines: Valeurs recommandées pour les farines. $4 p$.

Tabuc, 2007. Flore fongique de différents substrats et conditions optimales de production des mycotoxines. 190p.

Wagacha JM, Muthomi JW, 2007. Fusarium: Infection process, mechanisms of mycotoxin production and their role in pathogenesis in wheat. Crop Protection 26, 877-885. 